\title{
Estimating Parametric Line-Source Models With Electroencephalography
}

\author{
Nannan Cao*, Student Member, IEEE, İmam Şamil Yetik, Member, IEEE, Arye Nehorai, Fellow, IEEE, \\ Carlos H. Muravchik, Senior Member, IEEE, and Jens Haueisen, Member, IEEE
}

\begin{abstract}
We develop three parametric models for electroencephalography (EEG) to estimate current sources that are spatially distributed on a line. We assume a realistic head model and solve the EEG forward problem using the boundary element method (BEM). We present the models with increasing degrees of freedom, provide the forward solutions, and derive the maximum-likelihood estimates as well as Cramér-Rao bounds of the unknown source parameters. A series of experiments are conducted to evaluate the applicability of the proposed models. We use numerical examples to demonstrate the usefulness of our line-source models in estimating extended sources. We also apply our models to the real EEG data of $\mathrm{N} 20$ response that is known to have an extended source. We observe that the line-source models explain the N20 measurements better than the dipole model.
\end{abstract}

Index Terms-Cramér-Rao bounds, EEG, extended source modeling.

\section{INTRODUCTION}

$\mathbf{E}$ LECTROENCEPHALOGRAPHY (EEG) is a noninvasive technique to analyze the spatial and temporal activities in the brain. It has a high temporal resolution on the order of a few microseconds and is applied in clinical practice (e.g., neurology and psychiatry) [1] as well as basic neuroscience (e.g., in the investigation of primary sensory and motor functions or in the analysis of cognition) [2]. The EEG inverse problem consists of inferring the locations and signals of the underlying neural activities from the electric potentials measured on the scalp. It is ill-posed, and prior constraints need to be applied to obtain a unique solution [3].

Choosing an appropriate source model is an important step in solving the inverse problem. Most often, it is assumed that the source is small compared with its distances to the sensors and thus a current dipole is used to model it [4], [5]. Clearly, this approach is valid only if the electric activity is confined to a

Manuscript received July 20, 2005; revised May 6, 2006. This work was supported by the National Science Foundation (NSF) under Grant CCR-0105334. Asterisk indicates corresponding author.

*N. Cao is with the Department of Electrical and Systems Engineering, Washington University in St. Louis, MO 63130 USA. She is also with the Bryan 201, Campus Box 1127, 1 Brookings Drive, St. Louis, MO 63130 USA (e-mail: ncao4@ese.wustl.edu).

İ.Ş. Yetik is with the Department of Electrical and Computer Engineering, Illinois Institute of Technology, Chicago, IL 60616 USA.

A. Nehorai is with the Department of Electrical and Systems Engineering, Washington University in St. Louis, MO 63130 USA.

C. H. Muravchik is with the Departamento de Electrotecnia, Facultad de Ingenieria, Universidad Nacional de La Plata, Argentina.

J. Haueisen is with the Neurological University Hospital, Jena D-07743, Germany and also with the Institute of Biomedical Engineering and Informatics, TU-Ilmenau, Ilmenau, Germany.

Digital Object Identifier 10.1109/TBME.2006.880885 very small area. Multiple dipoles might be useful for modeling more separated and individually concentrated sources [6], [7], where it is critical to obtain a correct estimate of the number of sources, and the estimation performance will degrade if the electric activities are spread over a large area [3].

In many cases, the true sources are spatially distributed, and an extended source model is necessary to interpret the measurements more precisely. One approach is to use distributed source models and reconstruct the brain activities on a 3-D grid where each point is considered as a possible location of a current dipole source, therefore the restriction on the number of dipoles can be removed [8], [9]. However, there are two major problems with this approach. First, it is highly underdetermined: an infinite number of source distributions can lead to exactly the same potential map; and second, it has a high computational cost [3]. The ill-posed problem can be tackled by using regularization techniques [9], [10] and iterative focalization approaches [11], [12].

Another important issue with solving the EEG inverse problem is the selection of a proper head model, which affects the computation of induced potentials on the scalp. The simplest model is the homogeneous spherical head model, where the head is considered as a sphere with uniform conductivity [13]. This approach allows an analytical solution to the EEG forward problem and thus is computationally efficient, but it results in low source localization accuracy. The multishell spherical model can improve the performance by considering the head as several concentric shells with different conductivity values for the brain, skull, cerebrosphinal fluid, and scalp [14]-[16]. However, this model is still an oversimplification of the real head, and the localized source can not be coregistered to the brain anatomy precisely [17]. Much progress has been made recently in developing a realistic head model (RHM), where the head is modeled as multiple layers with different conductivities, and each inter-layer surface is obtained from head cross-section images from magnetic resonance imaging (MRI) or X-ray computerized tomography (CT) systems [5], [18]. Several comparative studies have shown that more accurate forward models can be achieved by RHM [19]-[21].

In this paper, we present three parametric line-source models for EEG assuming a realistic head model. We note that although we name our methods "line-source modeling," the real sources in the brain are not necessarily distributed exactly on a line. Nevertheless, we aim to show that electric sources which are more extended in one dimension could be better approximated by line-like models, and the spatial extent is a significant property of the source which should be considered in the modeling. One-dimensional source distribution was modeled 


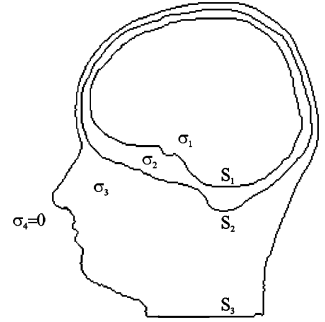

Fig. 1. Sketch of a realistic head model with $M=3$ isotropic conductivity layers, where $\sigma_{m}^{-}$and $\sigma_{m}^{+}$denote the conductivities of the layers inside and outside $S_{m}$ respectively $\left(\sigma_{m}^{+}=\sigma_{m+1}^{-}\right)$.

for MEG using current multipole expansion [22] or parametric source models [23]. The authors of both papers applied their approaches to the primary cortical response ("N20") to electric median nerve stimulation and showed improved estimation accuracy over merely utilizing the dipole source model. Our current work extends the ideas in [23] to EEG and incorporates a new model that is more general than the one in [23]. This extension is useful since EEG systems are less expensive and more commonly used than MEG; there are clinical applications where the MEG measurements are not available; and combined with the methods in [23], our models can be used to improve the estimation performance for simultaneous EEG/MEG recordings. We use the boundary element method (BEM) to solve the quasi-static Maxwell equations and formulate the EEG forward problem in a kernel-matrix form [24]. We estimate the source parameters using the maximum-likelihood (ML) method and derive the Cramér-Rao bound (CRB) to evaluate the estimation performance [25], [26]. The CRB provides a lower bound on the variance of any unbiased estimator. It is independent of the estimation algorithm thus provides the best estimation accuracy that can be expected from a certain model. We also compare the performances of the proposed line-source models and the dipole model in terms of the mean-squared error (MSE) and the Akaike's information criterion (AIC). AIC is a measure of model fitness which accounts for the trade-off between model complexity and accuracy [27], [28].

This paper is organized as follows. In Section II, we give a brief review of the RHM and BEM. In Section III we describe the line-source models with increasing degrees of freedom, formulate the EEG forward model, and derive the ML estimates of the unknown parameters. We discuss the CRBs in Section IV and give numerical examples in Section V; using both simulated data and real EEG measurements of N20 responses. Conclusions and future work are discussed in Section VI.

\section{Realistic Head Model and Boundary ELEMENT METHOD}

In the realistic head model, the head is considered to be a volume conductor $\mathcal{V}$ of $M$ homogeneous and isotropic layers separated by closed surfaces $S_{m}, m=1, \ldots, M$. The layers represent the scalp, skull, cerebro-spinal fluid, gray and white matter in the brain and are assumed to be immersed in an infinite homogeneous layer of zero conductivity, [18], [21]. See Fig. 1 for an example with $M=3$.

We need to use numerical methods such as the boundary element method (BEM) [29] or the finite element method (FEM)
[30] to solve the EEG forward problem with a realistic head model. Here, we utilize BEM and compute the solution in the following steps. We first pose the quasi-static Maxwell's equations as 2-D integral equations on all boundaries and then tessellate each boundary into small triangular elements, producing a set of nodes on each surface. We approximate the electric potentials by a linear combination of basis functions and solve the boundary integrals using the weighted residual technique. The whole procedure leads to a system of linear equations whose unknowns are the expansion coefficients. We solve for the coefficients and use them to interpolate the potentials at the EEG sensor positions. Below we briefly summarize the results; see [5], [18] for a detailed derivation.

Assuming an EEG sensor array with $m_{\mathrm{E}}$ electrodes and a total number of $N$ nodes on all boundaries, the electrical potentials at sensor positions, denoted by $\boldsymbol{v}_{\mathrm{E}}$, can be expressed as [5], [18]

$$
\boldsymbol{v}_{\mathrm{E}}=H_{\mathrm{E}}(D+H)^{\dagger} \boldsymbol{\phi}_{0}
$$

where $\boldsymbol{v}_{\mathrm{E}}$ is an $m_{\mathrm{E}} \times 1$ vector, $D$ and $H$ are $N \times N$ matrices, and $H_{\mathrm{E}}$ is of dimension $m_{\mathrm{E}} \times N$. Each entry of $D, H$, and $H_{\mathrm{E}}$ is a surface integral on a certain tessellation element, expressed as a function of the basis functions used to expand the potential field and the weighting functions used by the method of weighted residuals. The symbol " $\dagger$ " denotes pseudo-inverse, and the $N \times 1$ vector $\phi_{0}$ represents the electrical potentials at the node points, assuming the same source but immersed in an infinite homogeneous medium with conductivity $1 \Omega^{-1} \mathrm{~m}^{-1}$. Denoting the $i$ th element of $\boldsymbol{\phi}_{0}$ by $\boldsymbol{\phi}_{0}^{(i)}$, which corresponds to the potential at a node with position $\boldsymbol{r}_{i}$, we have

$$
\phi_{0}^{(i)}=\frac{1}{4 \pi} \int_{\mathcal{V}} \boldsymbol{J}\left(\boldsymbol{r}^{\prime}\right) \cdot \frac{\left(\boldsymbol{r}_{i}-\boldsymbol{r}^{\prime}\right)}{\left\|\boldsymbol{r}_{i}-\boldsymbol{r}^{\prime}\right\|^{3}} d^{3} r^{\prime}
$$

where $\boldsymbol{J}(\boldsymbol{r})$ represents the current source density and $d^{3} r^{\prime}$ is a differential volume element of $\mathcal{V}$. In particular, for a dipole source at position $\boldsymbol{p}$ with moment $\boldsymbol{q}$, i.e., $\boldsymbol{J}(\boldsymbol{r})=\boldsymbol{q} \delta(\boldsymbol{r}-\boldsymbol{p})$

$$
\boldsymbol{\phi}_{0}^{(i)}=\frac{1}{4 \pi} \frac{\boldsymbol{q} \cdot\left(\boldsymbol{r}_{i}-\boldsymbol{p}\right)}{\left\|\boldsymbol{r}_{i}-\boldsymbol{p}\right\|^{3}} .
$$

Note that the source parameters appear only in the vector $\phi_{0}$ in (2.1), and all the other matrices depend on the head geometry, conductivities, and sensor configurations. This structure will be utilized in Section III to construct the EEG forward model for the line sources in a kernel-matrix form and to simplify the calculation of the CRB.

\section{Source AND MEASuREment Models}

We present three parametric line-source models for EEG with increasing degrees of freedom, assuming a realistic head model. Considering $K$ independent trials and $N_{\mathrm{t}}$ time samples, the measured potentials at time $t$ in the $k$ th trial can be written as

$$
\begin{aligned}
\boldsymbol{y}_{k}(t) & =A\left(\boldsymbol{\theta}_{\mathrm{p}}\right) \boldsymbol{m}(t)+\boldsymbol{e}_{k}(t), \\
t & =1, \ldots, N_{\mathrm{t}}, \quad k=1, \ldots, K
\end{aligned}
$$


where the vector $\boldsymbol{\theta}_{\mathrm{p}}$ represents the source location parameters, $\boldsymbol{m}(t)$ the moment parameters, $\boldsymbol{y}_{k}(t)$ the measurements, $A\left(\boldsymbol{\theta}_{\mathrm{p}}\right)$ the array response matrix (also called lead field matrix), and $\boldsymbol{e}_{k}(t)$ the additive noise. The terms $A\left(\boldsymbol{\theta}_{\mathrm{p}}\right), \boldsymbol{\theta}_{\mathrm{p}}$, and $\boldsymbol{m}(t)$ assume different expressions for different line-source models as we describe in Sections III-A-C.

We use the maximum-likelihood method to estimate the source parameters $\boldsymbol{\theta}_{\mathrm{p}}$ and $\boldsymbol{m}(t)$. Assuming zero-mean Gaussian noise that is spatially and temporarily uncorrelated, the maximum-likelihood estimate (MLE) of $\boldsymbol{\theta}_{\mathrm{p}}$ is [4], [23]

$$
\hat{\boldsymbol{\theta}}_{\mathrm{p}}=\arg \min _{\boldsymbol{\theta}_{\mathrm{p}}} \sum_{t=1}^{N_{\mathrm{t}}}-\overline{\boldsymbol{y}}(t)^{T} P\left(\boldsymbol{\theta}_{\mathrm{p}}\right) \overline{\boldsymbol{y}}(t)
$$

where

$$
\begin{aligned}
\overline{\boldsymbol{y}}(t) & =\frac{1}{K} \sum_{k=1}^{K} \boldsymbol{y}_{k}(t) \\
P\left(\boldsymbol{\theta}_{\mathrm{p}}\right) & =A\left(\boldsymbol{\theta}_{\mathrm{p}}\right)\left[A\left(\boldsymbol{\theta}_{\mathrm{p}}\right)^{T} A\left(\boldsymbol{\theta}_{\mathrm{p}}\right)\right]^{-1} A\left(\boldsymbol{\theta}_{\mathrm{p}}\right)^{T}
\end{aligned}
$$

and the MLE of $\boldsymbol{m}(t)$ is

$$
\hat{\boldsymbol{m}}(t)=\left[A\left(\hat{\boldsymbol{\theta}}_{\mathrm{p}}\right)^{T} A\left(\hat{\boldsymbol{\theta}}_{\mathrm{p}}\right)\right]^{-1} A\left(\hat{\boldsymbol{\theta}}_{\mathrm{p}}\right)^{T} \overline{\boldsymbol{y}}(t) .
$$

Note that it is possible to consider more complex noise models such as spatially correlated [4] or spatiotemporally correlated [31] Gaussian noise models, but we are not going to explore that aspect in this paper since our main focus is on the line-source modeling.

In the following, we describe in detail the proposed line source models and in particular the EEG forward models corresponding to each one.

\section{A. Constant-Radius Constant-Moment (CRCM) Model}

Here the source position is an arc with an arbitrary orientation on a spherical surface: it has a fixed distance from the head center, its azimuth varies within a certain interval, and its elevation changes linearly with the azimuth. The source moment is assumed to be uniformly distributed along the arc; see Fig. 2(a). This model is an extension of the VACM model used for MEG in [23], where the source orientation is fixed along the elevation direction (corresponding to $\rho=0$ ). Using spherical coordinates with $p$ representing the distance from the center, $\xi$ the elevation, and $\varphi$ the azimuth, we have

$$
\begin{aligned}
\boldsymbol{J}(\boldsymbol{r}, t)= & \boldsymbol{q}(t) \delta\left(p-p_{0}\right) \delta\left(\xi-\xi_{0}-\rho \varphi\right) \\
& \times\left[u\left(\varphi-\varphi_{1}\right)-u\left(\varphi-\varphi_{2}\right)\right] \\
\boldsymbol{q}(t)= & {\left[q_{x}(t), q_{y}(t), q_{z}(t)\right]^{T} }
\end{aligned}
$$

where $p_{0}$ is the fixed radius, $\varphi_{1}$ and $\varphi_{2}$ the azimuth limits, and $\xi_{0}$ the constant elevation. The slope $\rho$ determines the source orientation, and $u(\varphi)$ is the unit step function defined as $u(\varphi)=$ 1 for $\varphi>0$ and $u(\varphi)=0$ for $\varphi \leq 0$. Thus, in this model the unknown position parameter vector is $\boldsymbol{\theta}_{\mathrm{p}}=\left[p_{0}, \xi_{0}, \rho, \varphi_{1}, \varphi_{2}\right]^{T}$.
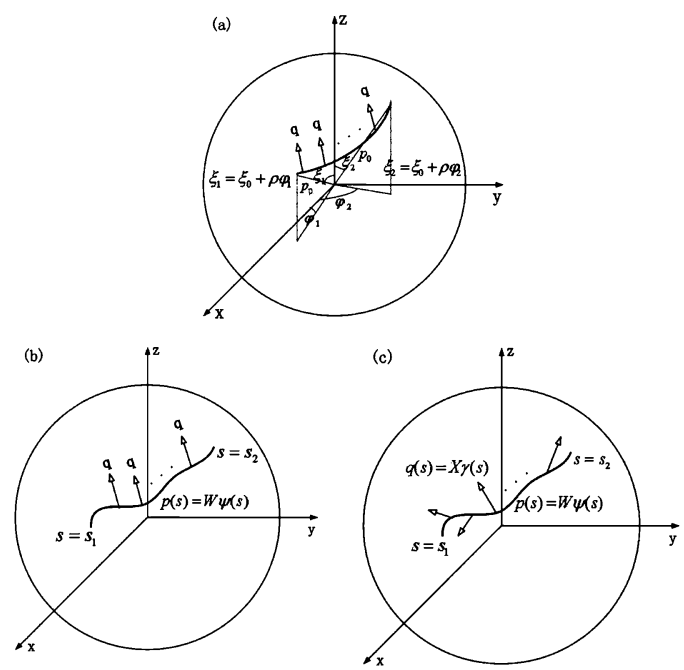

Fig. 2. Illustration of the three line-source models. (a) CRCM model, (b) VPCM model, and (c) VPVM model. See Section III for more details.

Utilizing the sifting property of the delta function, (2.2) can be written as

$$
\begin{aligned}
& \boldsymbol{\phi}_{0}^{(i)}=\boldsymbol{v}\left(\boldsymbol{r}_{i}, \boldsymbol{\theta}_{\mathrm{p}}\right) \boldsymbol{q}(t) \\
& =\left[\begin{array}{c}
\int_{\varphi_{1}}^{\varphi_{2}} \frac{\left(r_{i x}-p_{0} \sin \left(\xi_{0}+\rho \varphi\right) \cos \varphi\right) p_{0} \sin \left(\xi_{0}+\rho \varphi\right)}{4 \pi \nu\left(\boldsymbol{r}_{i}, p_{0}, \xi_{0}, \rho, \varphi\right)} d \varphi \\
\int_{\varphi_{1}}^{\varphi_{2}} \frac{\left(r_{i y}-p_{0} \sin \left(\xi_{0}+\varphi\right) \sin \varphi p_{0} \sin \left(\xi_{0}+\rho \varphi\right)\right.}{4 \pi \nu\left(\boldsymbol{r}_{i}, p_{0}, \xi_{0}, \rho, \varphi\right)} d \varphi \\
\int_{\varphi_{1}}^{\varphi_{2}} \frac{\left(r_{i z}-p_{0} \cos \left(\xi_{0}+\rho \varphi\right)\right) p_{0} \sin \left(\xi_{0}+\rho \varphi\right)}{4 \pi \nu\left(\boldsymbol{r}_{i}, p_{0}, \xi_{0}, \rho, \varphi\right)} d \varphi
\end{array}\right]^{T} \boldsymbol{q}(t)
\end{aligned}
$$

where

$$
\begin{aligned}
\nu\left(\boldsymbol{r}_{i}, p_{0}, \xi_{0}, \rho, \varphi\right)=[ & \left(r_{i x}-p_{0} \sin \left(\xi_{0}+\rho \varphi\right) \cos \varphi\right)^{2} \\
& +\left(r_{i y}-p_{0} \sin \left(\xi_{0}+\rho \varphi\right) \sin \varphi\right)^{2} \\
& \left.+\left(r_{i z}-p_{0} \cos \left(\xi_{0}+\rho \varphi\right)\right)^{2}\right]^{3 / 2}
\end{aligned}
$$

and $\boldsymbol{r}_{i}=\left[r_{i x}, r_{i y}, r_{i z}\right]^{T}$ is the position of the $i$ th node. Defining an $N \times 3$ matrix $V\left(\boldsymbol{\theta}_{\mathrm{p}}\right)$ as

$$
V\left(\boldsymbol{\theta}_{\mathrm{p}}\right)=\left[\boldsymbol{v}\left(\boldsymbol{r}_{1}, \boldsymbol{\theta}_{\mathrm{p}}\right)^{T}, \boldsymbol{v}\left(\boldsymbol{r}_{2}, \boldsymbol{\theta}_{\mathrm{p}}\right)^{T}, \ldots, \boldsymbol{v}\left(\boldsymbol{r}_{N}, \boldsymbol{\theta}_{\mathrm{p}}\right)^{T}\right]^{T} .
$$

Equation (2.1) becomes

$$
\boldsymbol{v}_{\mathrm{E}}(t)=H_{\mathrm{E}}(D+H)^{\dagger} V\left(\boldsymbol{\theta}_{\mathrm{p}}\right) \boldsymbol{q}(t)
$$

and the measured EEG potentials can be expressed as

$$
\begin{aligned}
\boldsymbol{y}_{k}(t) & =\boldsymbol{v}_{\mathrm{E}}(t)+\boldsymbol{e}_{k}(t) \\
& =\underbrace{H_{\mathrm{E}}(D+H)^{\dagger} V\left(\boldsymbol{\theta}_{\mathrm{p}}\right)}_{A\left(\theta_{\mathrm{p}}\right)} \underbrace{\boldsymbol{q}(t)}_{m(t)}+\boldsymbol{e}_{k}(t) .
\end{aligned}
$$

Equation (3.12) shows that the EEG forward model can be written in the form of (3.4). For this particular case $A\left(\boldsymbol{\theta}_{\mathrm{p}}\right)=$ $H_{\mathrm{E}}(D+H)^{\dagger} V\left(\boldsymbol{\theta}_{\mathrm{p}}\right)$ and $\boldsymbol{m}(t)=\boldsymbol{q}(t)=\left[q_{x}(t), q_{y}(t), q_{z}(t)\right]^{T}$.

\section{B. Variable-Position Constant-Moment (VPCM) Model}

This model provides more degrees of freedom for the source position than the CRCM model: the source position is allowed 
to be a parametric curve in 3-D space instead of an arc on a spherical surface; see Fig. 2(b). We represent the source position in Cartesian coordinates as

$$
p(s)=\left[p_{x}(s), p_{y}(s), p_{z}(s)\right]^{T}, \quad s \in\left[s_{1}, s_{2}\right]
$$

where $s$ is the curve parameter with limits $s_{1}$ and $s_{2}$. Accordingly, the source current density becomes

$$
\begin{aligned}
\boldsymbol{J}(\boldsymbol{r}, t) & = \begin{cases}\boldsymbol{q}(t), & \boldsymbol{r}=\left[p_{x}(s), p_{y}(s), p_{z}(s)\right]^{T}, s \in\left(s_{1}, s_{2}\right) \\
0, & \text { elsewhere }\end{cases} \\
\boldsymbol{q}(t) & =\left[q_{x}(t), q_{y}(t), q_{z}(t)\right]^{T}
\end{aligned}
$$

We assume the spatial distribution of the source position $\boldsymbol{p}(s)$ can be described by a linear combination of basis functions as

$$
\boldsymbol{p}(s)=W \boldsymbol{\psi}(s)
$$

where $\boldsymbol{\psi}(s)=\left[\psi_{1}(s), \psi_{2}(s), \ldots, \psi_{d}(s)\right]^{T}$ is a $d \times 1$ vector of known basis functions, and $W$ is a $3 \times d$ matrix of unknown coefficients [4], [23]. This parametrization allows us to exploit the prior information and reduce the number of unknown parameters. Substituting (3.14)-(3.16) into (2.1) and (2.2), we have

$$
\boldsymbol{y}_{k}(t)=\underbrace{H_{\mathrm{E}}(D+H)^{\dagger} V\left(\boldsymbol{\theta}_{\mathrm{p}}\right)}_{A\left(\theta_{\mathrm{p}}\right)} \underbrace{\boldsymbol{q}(t)}_{m(t)}+\boldsymbol{e}_{k}(t)
$$

where $\boldsymbol{\theta}_{\mathrm{p}}=\left[s_{1}, s_{2}, \operatorname{vec}^{T}(W)\right]^{T}$, the operator "vec" transforms a matrix into a column vector by stacking its columns on top of each other, $\boldsymbol{m}(t)=\boldsymbol{q}(t)=\left[q_{x}(t), q_{y}(t), q_{z}(t)\right]^{T}$, and the $i$ th row of the $N \times 3$ matrix $V\left(\boldsymbol{\theta}_{\mathrm{p}}\right)$ is

$$
\begin{aligned}
\boldsymbol{v}\left(\boldsymbol{r}_{i}, \boldsymbol{\theta}_{\mathrm{p}}\right)=\frac{1}{4 \pi} & {\left[\begin{array}{l}
\int_{s_{1}}^{s_{2}} \sum_{\tau=1}^{d}\left(r_{i x}-W_{1 \tau} \psi_{\tau}(s)\right) \frac{\alpha(\boldsymbol{\psi}(s))}{\beta\left(\boldsymbol{\psi}(s), \boldsymbol{r}_{i}\right)} d s \\
\int_{s_{1}}^{s_{2}} \sum_{\tau=1}^{d}\left(r_{i y}-W_{2 \tau} \psi_{\tau}(s)\right) \frac{\alpha(\boldsymbol{\psi}(s))}{\beta\left(\boldsymbol{\psi}(s), \boldsymbol{r}_{i}\right)} d s \\
\int_{s_{1}}^{s_{2}} \sum_{\tau=1}^{d}\left(r_{i z}-W_{3 \tau} \psi_{\tau}(s)\right) \frac{\alpha(\boldsymbol{\psi}(s))}{\beta\left(\boldsymbol{\psi}(s), \boldsymbol{r}_{i}\right)} d s
\end{array}\right]^{T} }
\end{aligned}
$$

with

$$
\begin{aligned}
\alpha(\boldsymbol{\psi}(s))= & \sqrt{p_{x}^{\prime}(s)^{2}+p_{y}^{\prime}(s)^{2}+p_{z}^{\prime}(s)^{2}} \\
\beta\left(\boldsymbol{\psi}(s), \boldsymbol{r}_{i}\right)= & {\left[\left(r_{i x}-p_{x}(s)\right)^{2}+\left(r_{i y}-p_{y}(s)\right)^{2}\right.} \\
& \left.+\left(r_{i z}-p_{z}(s)\right)^{2}\right]^{3 / 2}
\end{aligned}
$$

The superscript " "'” denotes the first derivative with respect to $s$, and $\boldsymbol{r}_{i}=\left[r_{i x}, r_{i y}, r_{i z}\right]^{T}$ is the position of the $i$ th node.

\section{Variable-Position Variable-Moment (VPVM) Model}

This is the most general model: the source position consists of a parametric curve and the source moment is allowed to vary along the position; see Fig. 2(c). Hence, the current density is

$$
\begin{aligned}
& \boldsymbol{J}(\boldsymbol{r}, t)=
\end{aligned}
$$

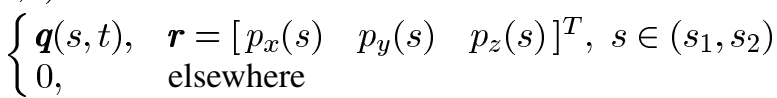

$$
\begin{aligned}
& \boldsymbol{q}(s, t)=\left[q_{x}(s, t), q_{y}(s, t), q_{z}(s, t)\right]^{T} .
\end{aligned}
$$

We describe the spatial variation of the moment density $\boldsymbol{q}(s, t)$ using basis functions as well

$$
\boldsymbol{q}(s, t)=X(t) \boldsymbol{\gamma}(s)
$$

where $\gamma(s)=\left[\gamma_{1}(s), \gamma_{2}(s), \ldots, \gamma_{l}(s)\right]^{T}$ is an $l \times 1$ vector of known basis functions, and $X(t)$ is a $3 \times l$ matrix of time varying unknown coefficients. Substituting (3.16), (3.20), and (3.22) into (2.1) and (2.2), we have

$$
\boldsymbol{y}_{k}(t)=\underbrace{H_{\mathrm{E}}(D+H)^{\dagger} V\left(\boldsymbol{\theta}_{\mathrm{p}}\right)}_{A\left(\theta_{\mathrm{p}}\right)} \underbrace{\operatorname{vec}(X(t))}_{m(t)}+\boldsymbol{e}_{k}(t)
$$

where $\boldsymbol{\theta}_{\mathrm{p}}=\left[s_{1}, s_{2}, \operatorname{vec}^{T}(W)\right]^{T}, \boldsymbol{m}(t)=\operatorname{vec}(X(t))$, and $V\left(\boldsymbol{\theta}_{\mathrm{p}}\right)$ is an $N \times 3 l$ matrix

$$
V\left(\boldsymbol{\theta}_{\mathrm{p}}\right)=\left[V_{1}\left(\boldsymbol{\theta}_{\mathrm{p}}, \gamma_{1}(s)\right) V_{2}\left(\boldsymbol{\theta}_{\mathrm{p}}, \gamma_{2}(s)\right) \ldots V_{l}\left(\boldsymbol{\theta}_{\mathrm{p}}, \gamma_{l}(s)\right)\right] .
$$

The $i$ th row of the $j$ th submatrix $V_{j}\left(\boldsymbol{\theta}_{\mathrm{p}}, \gamma_{j}(s)\right)$ is

$\boldsymbol{v}\left(\boldsymbol{r}_{i}, \boldsymbol{\theta}_{\mathrm{p}}\right)=\frac{1}{4 \pi}$

$$
\times\left[\begin{array}{l}
\int_{s_{1}}^{s_{2}} \sum_{\tau=1}^{d}\left(r_{i x}-W_{1 \tau} \psi_{\tau}(s)\right) \frac{\alpha(\boldsymbol{\psi}(s)) \gamma_{j}(s)}{\beta\left(\boldsymbol{\psi}(s), \boldsymbol{r}_{i}\right)} d s \\
\int_{s_{1}}^{s_{2}} \sum_{\tau=1}^{d}\left(r_{i y}-W_{2 \tau} \psi_{\tau}(s)\right) \frac{\alpha(\boldsymbol{\psi}(s)) \gamma_{j}(s)}{\beta\left(\boldsymbol{\psi}(s), \boldsymbol{r}_{i}\right)} d s \\
\int_{s_{1}}^{s_{2}} \sum_{\tau=1}^{d}\left(r_{i z}-W_{3 \tau} \psi_{\tau}(s)\right) \frac{\alpha(\boldsymbol{\psi}(s)) \gamma_{j}(s)}{\beta\left(\boldsymbol{\psi}(s), \boldsymbol{r}_{i}\right)} d s
\end{array}\right]^{T}
$$

where $\alpha(\boldsymbol{\psi}(s))$ and $\beta\left(\boldsymbol{\psi}(s), \boldsymbol{r}_{i}\right)$ are defined in (3.18) and (3.19), respectively.

\section{Interrelationship Between the Models}

The proposed line-source models are related to each other as follows.

- CRCM is a special case of VPCM: VPCM becomes CRCM if we select the azimuth $\varphi$ as the curve parameter $s$ and let

$$
\begin{aligned}
\rho & =0 \\
W & =\left[\begin{array}{ccc}
0 & 0 & p_{0} \sin \xi_{0} \\
0 & p_{0} \sin \xi_{0} & 0 \\
p_{0} \cos \xi_{0} & 0 & 0
\end{array}\right] \\
\boldsymbol{\psi}(s) & =\left[\begin{array}{lll}
1 & \sin s & \cos s
\end{array}\right]^{T} .
\end{aligned}
$$

- VPCM is a special case of VPVM: VPVM becomes VPCM when $\boldsymbol{q}(s, t)=\boldsymbol{q}(t)$. 
- CRCM is a special case of VPVM: VPVM becomes CRCM if the conditions for the two special cases above are satisfied.

\section{E. Computational Issues}

We can see from (3.12), (3.17), and (3.23) that for all linesource models, the array response matrix $A\left(\boldsymbol{\theta}_{\mathrm{p}}\right)$ is in the form of

$$
A\left(\boldsymbol{\theta}_{\mathrm{p}}\right)=H_{\mathrm{E}}(D+H)^{\dagger} V\left(\boldsymbol{\theta}_{\mathrm{p}}\right)
$$

where the matrix $V\left(\boldsymbol{\theta}_{\mathrm{p}}\right)$ has different expressions for different source models.

A large proportion of the computational effort of calculating (3.27) and the MLEs of the source parameters (3.5)-(3.8) lies in computing 1) the entries of matrices $H_{\mathrm{E}}, D$, and $H$, which are surface integrals over certain tessellation areas; 2) the pseudoinverse of the matrix $(D+H)$, which depends on the number of nodes $N$. For the first part, there exist analytical formulae for rapid calculation of each matrix element if the surface is tessellated into triangles as is usually done [32]-[34]. Calculating the pseudoinverse can be time consuming if $N$ is very large (e.g., $N$ is around 4500 for the data we use.) However, since $D$ and $H$ depend only on the head geometry and conductivities, they need to be calculated only once for a certain subject regardless of the source parameters. Therefore, computing $(H+D)^{\dagger}$ will not pose a very big problem in the calculation of the MLE, which is a minimization procedure over all possible source parameters (see (3.5)). This separation will also be utilized for calculating the CRBs in Section IV.

\section{CRAmÉR-RAo BoundS}

The Cramér-Rao bound is a lower bound on the covariance of any unbiased estimator. It is independent of the algorithm used for the estimation, thus establishes a universal performance limit. It is an asymptotically tight bound under certain hypotheses; i.e., the bound can be achieved as the number of data samples becomes very large. For a certain problem, if the maximum-likelihood estimator exists, it can asymptotically achieve the CRB [25], [26].

Let $\boldsymbol{\theta}=\left[\boldsymbol{\theta}_{\mathrm{p}}^{T}, \boldsymbol{m}(1)^{T}, \ldots, \boldsymbol{m}\left(N_{\mathrm{t}}\right)^{T}\right]^{T}$ represent all the unknown source parameters, $\hat{\boldsymbol{\theta}}$ be an unbiased estimator of $\boldsymbol{\theta}$, and $\mathcal{I}(\boldsymbol{\theta})$ denote the Fisher information matrix (FIM). The Cramér-Rao inequality establishes that

$$
E\left\{(\boldsymbol{\theta}-\hat{\boldsymbol{\theta}})(\boldsymbol{\theta}-\hat{\boldsymbol{\theta}})^{T}\right\} \geq \operatorname{CRB}(\boldsymbol{\theta})=\mathcal{I}^{-1}(\boldsymbol{\theta})
$$

where the inequality sign states that the difference between the matrices on the left and right sides is positive semidefinite. Note that the diagonal elements of the matrix $\operatorname{CRB}(\boldsymbol{\theta})$ are particularly useful since they set bounds on the variance of the parameters.
For the measurement model (3.4), assuming zero-mean Gaussian noise with covariance matrix $\Sigma=\sigma_{\mathrm{E}}^{2} I_{m_{\mathrm{E}}}$, the Fisher information matrix is

$$
\begin{aligned}
\mathcal{I}(\boldsymbol{\theta}) & =\left[\begin{array}{ll}
\mathcal{I}_{\mathrm{pp}} & \mathcal{I}_{\mathrm{qp}}^{T} \\
\mathcal{I}_{\mathrm{qp}} & \mathcal{I}_{\mathrm{qq}}
\end{array}\right] \\
& =\left[\begin{array}{ccccc}
\mathcal{I}_{\mathrm{pp}} & \mathcal{I}_{\mathrm{qp}}^{T}(1) & \mathcal{I}_{\mathrm{qp}}^{T}(2) & \ldots & \mathcal{I}_{\mathrm{qp}}^{T}\left(N_{\mathrm{t}}\right) \\
\mathcal{I}_{\mathrm{qp}}(1) & \mathcal{I}_{\mathrm{qq}} & 0 & \ldots & 0 \\
\mathcal{I}_{\mathrm{qp}}(2) & 0 & \mathcal{I}_{\mathrm{qq}} & \ldots & 0 \\
& & \vdots & & \\
\mathcal{I}_{\mathrm{qp}}\left(N_{\mathrm{t}}\right) & 0 & 0 & \ldots & \mathcal{I}_{\mathrm{qq}}
\end{array}\right]
\end{aligned}
$$

where

$$
\begin{aligned}
\mathcal{I}_{\mathrm{pp}} & =\frac{K}{\sigma_{\mathrm{E}}^{2}} \sum_{t=1}^{N_{\mathrm{t}}} D_{A}^{T}\left(\boldsymbol{\theta}_{\mathrm{p}}\right)\left[\left(\boldsymbol{m}(t) \boldsymbol{m}^{T}(t)\right) \otimes I_{m_{\mathrm{E}}}\right] D_{A}\left(\boldsymbol{\theta}_{\mathrm{p}}\right) \\
\mathcal{I}_{\mathrm{qp}}(t) & =\frac{K}{\sigma_{\mathrm{E}}^{2}} A^{T}\left(\boldsymbol{\theta}_{\mathrm{p}}\right)\left(\boldsymbol{m}^{T}(t) \otimes I_{m_{\mathrm{E}}}\right) D_{A}\left(\boldsymbol{\theta}_{\mathrm{p}}\right) \\
\mathcal{I}_{\mathrm{qq}} & =\frac{K}{\sigma_{\mathrm{E}}^{2}} A^{T}\left(\boldsymbol{\theta}_{\mathrm{p}}\right) A\left(\boldsymbol{\theta}_{\mathrm{p}}\right)
\end{aligned}
$$

the matrix $I_{m_{\mathrm{E}}}$ is an $m_{\mathrm{E}} \times m_{\mathrm{E}}$ identity matrix, “ $\otimes$ " denotes the Kronecker product [35], and $D_{A}\left(\boldsymbol{\theta}_{\mathrm{p}}\right)$ is defined as [4], [23]

$$
D_{A}\left(\boldsymbol{\theta}_{\mathrm{p}}\right) \triangleq \frac{\partial \operatorname{vec}\left(A\left(\boldsymbol{\theta}_{\mathrm{p}}\right)\right)}{\partial \boldsymbol{\theta}_{\mathrm{p}}^{T}}
$$

Utilizing the structure of $A\left(\boldsymbol{\theta}_{\mathrm{p}}\right)$ in (3.27), we rewrite $D_{A}\left(\boldsymbol{\theta}_{\mathrm{p}}\right)$ as

$$
\begin{aligned}
D_{A}\left(\boldsymbol{\theta}_{\mathrm{p}}\right)= & \frac{\partial \operatorname{vec}\left(A\left(\boldsymbol{\theta}_{\mathrm{p}}\right)\right)}{\partial \boldsymbol{\theta}_{\mathrm{p}}^{T}} \\
= & \left(I_{n_{\mathrm{p}}} \otimes\left(H_{\mathrm{E}}(D+H)^{\dagger}\right)\right) \\
& \cdot \frac{\partial \operatorname{vec}\left(V\left(\boldsymbol{\theta}_{\mathrm{p}}\right)\right)}{\partial \boldsymbol{\theta}_{\mathrm{p}}^{T}}
\end{aligned}
$$

where $n_{\mathrm{p}}$ is the number of unknown position parameters and $I_{n_{\mathrm{p}}}$ is an $n_{\mathrm{p}} \times n_{\mathrm{p}}$ identity matrix. In this way, we need to calculate only the second part of (4.31) for any possible $\boldsymbol{\theta}_{\mathrm{p}}$, reducing the computational cost to obtain CRBs for a certain subject.

\section{NUMERICAL RESULTS}

We conducted a series of experiments to demonstrate the applicability of the proposed models in estimating the line sources. We used a three-layer realistic head model composed of the brain, skull, and scalp, assuming the conductivity values to be $0.33 \Omega^{-1} \mathrm{~m}^{-1}$ for the scalp and brain, and $0.0042 \Omega^{-1} \mathrm{~m}^{-1}$ for the skull [18], [24], [36]. The inter-layer surfaces were tessellated into a total of 9290 triangles (2884 on the brain, 3240 on the skull, and 3166 on the scalp) through MRI (Philips, Hamburg, Germany). We used 32 electrodes glued to the scalp of the subject (SynAmps, Compumedics Neuroscan, El Paso, USA), 
and they were placed close together over the stimulated hemisphere; see [37] for more details on electrode setup.

For the BEM, we used linear discretization, where the vertices of each triangle are regarded as node points [24], [32]-[34]. Let $\boldsymbol{r}_{1}, \boldsymbol{r}_{2}$, and $\boldsymbol{r}_{3}$ denote the three vertices of an arbitrary triangle on the $m$ th surface, ordered in such a way that the permutation $\boldsymbol{r}_{1} \rightarrow \boldsymbol{r}_{2} \rightarrow \boldsymbol{r}_{3}$ corresponds to the right-hand rule to the outward normal of the surface; the discretization consists of 1) choosing the weighting function in a collocation form, i.e., $w_{i}^{m}=\delta(\boldsymbol{r}-$ $\left.\boldsymbol{r}_{i}^{m}\right), i=1,2,3 ; 2$ ) defining the basis functions for potentials as [5]

$$
\begin{aligned}
h_{1}^{m}(\boldsymbol{r}) & =\frac{\boldsymbol{r} \cdot\left(\boldsymbol{r}_{2} \times \boldsymbol{r}_{3}\right)}{\boldsymbol{r}_{1} \cdot\left(\boldsymbol{r}_{2} \times \boldsymbol{r}_{3}\right)} \\
h_{2}^{m}(\boldsymbol{r}) & =\frac{\boldsymbol{r} \cdot\left(\boldsymbol{r}_{3} \times \boldsymbol{r}_{1}\right)}{\boldsymbol{r}_{1} \cdot\left(\boldsymbol{r}_{2} \times \boldsymbol{r}_{3}\right)} \\
h_{3}^{m}(\boldsymbol{r}) & =\frac{\boldsymbol{r} \cdot\left(\boldsymbol{r}_{1} \times \boldsymbol{r}_{2}\right)}{\boldsymbol{r}_{1} \cdot\left(\boldsymbol{r}_{2} \times \boldsymbol{r}_{3}\right)}
\end{aligned}
$$

where $\boldsymbol{r}$ corresponds to a position inside the triangle, "." denotes the inner product, and " $\times$ " the cross product. Linear interpolation provides better accuracy in EEG forward modeling [34] than the "center of gravity" (COG) method used in [18].

\section{A. Numerical Results Using Simulated EEG Data}

We first present the results using simulated EEG data. We compared the performances of different source models and calculated the Cramér-Rao bounds for the CRCM model. Throughout the experiments in this subsection, we selected the noise variance to obtain a signal-to-noise ratio (SNR) of 20 $\mathrm{dB}$ [36]. We define SNR as SNR $=10 \log \left(\left(\sum_{i=1}^{m_{\mathrm{E}}} s_{i}^{2}\right) / \sigma_{\mathrm{E}}^{2}\right)$, where $s_{i}^{2}=1 / N_{\mathrm{t}} \sum_{t=1}^{N_{\mathrm{t}}} v_{\mathrm{Ei}}^{2}(t)$ is the signal power at the $i$ th sensor.

1) Comparison of Different Models: We assumed two types of source distributions and estimated the source parameters using the proposed line-source models and the dipole source model. We analyzed the estimation accuracy and model fitness using the mean-squared error (MSE) and the Akaike's information criterion (AIC) [27], [28]. The AIC penalizes the $\log$-likelihood function for additional source parameters, and hence accounts for the tradeoff between model complexity and accuracy. It is defined as

$$
\mathrm{AIC}=-2 \ln \left(L_{\theta}(\boldsymbol{y})\right)+2 g
$$

where $L_{\theta}(\boldsymbol{y})$ is the likelihood function and $g$ is the number of unknown parameters. For normally distributed noise with variance $\sigma^{2}$

$$
\mathrm{AIC}=\ln \left((2 \pi)^{m N_{\mathrm{t}} K} \sigma^{2}\right)+\frac{\sum_{t=1}^{N_{\mathrm{t}}} \sum_{j=1}^{K} \boldsymbol{e}_{j}^{T}(t) \boldsymbol{e}_{j}(t)}{\sigma^{2}}+2 g
$$

where $\boldsymbol{e}_{j}(t)$ is the noise at the $t$ th time sample in the $j$ th trial. Hence, a smaller AIC value indicates a better fit of the model.

2) Example 1: We used a line source with a fixed distance from the center $p_{0}=85 \mathrm{~mm}$, a fixed elevation $\xi_{0}=45^{\circ}, \rho=0$,
TABLE I

Comparison of Estimation Performance Using Simulated Data. The Source Position Is Assumed to Be AN ARC ON A SPHERE With $p_{0}=85 \mathrm{~mm}, \xi_{0}=45^{\circ}, \varphi_{1}=20^{\circ}$, AND $\varphi_{2}=60^{\circ}$. THE SOURCE MOMENT DENSITY Is CHOSEN To BE $q_{x}=q_{y}=q_{z}=150 \mathrm{nA}$

\begin{tabular}{|c|c|c|c|}
\hline Source model & No. of parameters & MSE $\left(\mu V^{2}\right)$ & AIC \\
\hline Dipole & 6 & 1.91 & 97.13 \\
CRCM & 8 & 1.21 & 90.26 \\
VPCM & 9 & 1.35 & 94.89 \\
VPVM & 15 & 1.79 & 113.36 \\
\hline
\end{tabular}

and varying azimuth with limits $\varphi_{1}=20^{\circ}$ and $\varphi_{2}=60^{\circ}$. We chose the moment density as $q_{x}=q_{y}=q_{z}=150 \mathrm{nA}$, and applied the CRCM model to generate the electric potentials.

We estimated the source parameters using the proposed linesource models as well as the focal dipole model. For both VPCM and VPVM models, we chose basis functions $\psi(s)=[1, s]^{T}$ to approximate the source position, and $\gamma(s)=\left[1, s, s^{2}\right]^{T}$ was used to model the moment variation in the VPVM model.

The simulation results are shown in Table I. We observe that the MSEs of all the estimated line-source models are smaller than that of the dipole source model, showing that the linesource models can explain the data better than the focal one if the real source is extended sufficiently. Note that the VPCM model has a higher MSE than the CRCM model even though it is more general. The reason is that the estimation performance of the VPCM parameters is closely related to the choice of basis functions (see (3.16)), and in this case we used first order polynomials to approximate a real source which lies on a spherical surface. Choosing higher order polynomials could reduce the MSE, but the AIC value would certainly increase. Therefore, considering the tradeoff between the model complexity and estimation accuracy, we think it enough to stay with first order polynomials. More discussion about how to choose basis functions for the real data is given in Section VI.

The effectiveness of our line-source models in improving the estimation performance is supported by the AIC values as well (see the last column of Table I): both the CRCM and VPCM models have smaller AICs than the dipole model. The VPVM model has a larger AIC in this case due to the uniform distribution of the source moment density we chose. The advantage of the VPVM model to model the spatially varying moment density is demonstrated by the second example below.

3) Example 2: In this example, the source position is a straight line between $[x, y, z]=[12,15,60] \mathrm{mm}$ and $[x, y, z]=[40,40,52] \mathrm{mm}$. It has a length of $38 \mathrm{~mm}$ and a larger change in the elevation than the azimuth. We chose the moment density $\boldsymbol{q}(s)=\left[q_{x}(s), q_{y}(s), q_{z}(s)\right]^{T}$ with $q_{x}=-s^{2}+400 \mathrm{nA}$, $q_{y}=200 \mathrm{nA}$, and $q_{z}=-s^{2}+2 s+300 \mathrm{nA}$, so that it is small at the ends and large at the center.

We produced the EEG data using the VPVM model and estimated the source parameters with the same basis functions as in Example 1; see Table II. We observe that all the line-source models have smaller MSEs and AICs than the dipole model.

These examples show that the line-source models fit the data better than the dipole model if the real source extends over a sufficiently large range. The VPVM model is useful to capture the spatial variation of the moment density even though the large number of unknown parameters makes it less attractive in 
TABLE II

Comparison of Estimation Performance Using Simulated Data. THE SOURCE Is ASSUMED TO LIE ON A STRAIGHT LINE BETwEEN $[12,15,60] \mathrm{mm}$ AND $[40,40,52] \mathrm{mm}$. THE Source Moment Density Is Chosen to BE $q_{x}=-s^{2}+400 \mathrm{nA}, q_{y}=200 \mathrm{nA}, \mathrm{AND} q_{z}=-s^{2}+2 s+300 \mathrm{nA}$, WHERE $s$ IS THE CURVE PARAMETER

\begin{tabular}{|c|c|c|c|}
\hline Source model & No. of parameters & MSE $\left(\mu V^{2}\right)$ & AIC \\
\hline Dipole & 6 & 1.65 & 108.77 \\
CRCM & 8 & 1.32 & 103.16 \\
VPCM & 9 & 0.91 & 96.27 \\
VPVM & 15 & 0.63 & 101.29 \\
\hline
\end{tabular}
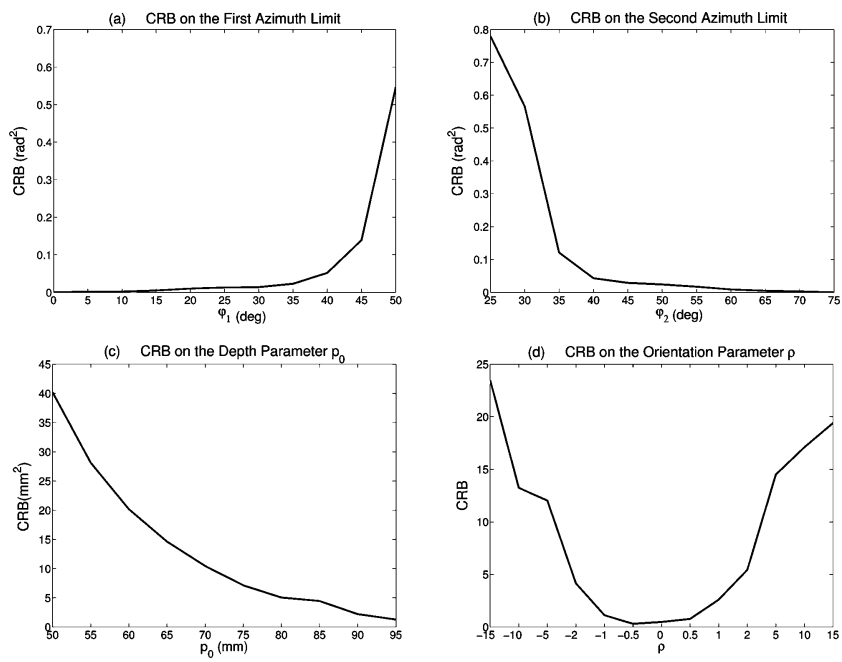

Fig. 3. CRBs on the unknown source position parameters for the CRCM model $(\mathrm{SNR}=20 \mathrm{~dB})$. (a) $p_{0}=85 \mathrm{~mm}, \xi_{0}=45^{\circ}, \rho=0$, and $\varphi_{2}=60^{\circ}$; (b) $p_{0}=85 \mathrm{~mm}, \xi_{0}=45^{\circ}, \rho=0$, and $\varphi_{1}=20^{\circ}$; (c) $\xi_{0}=45^{\circ}, \rho=0$, $\varphi_{1}=20^{\circ}$, and $\varphi_{2}=60^{\circ}$; (d) $p_{0}=85 \mathrm{~mm}$ and fixed source length.

some cases. We also observe during the simulations that the estimated dipole source is always in a neighborhood of the line source. That is, the estimated dipole location can be used to approximate the center of mass of the actual source distribution. Although this is a result from computer simulations, it is intuitively appealing and is helpful for the initialization of the ML method when estimating the source parameters with real EEG measurements.

4) Cramér-Rao Bound Results: We computed the Cramér-Rao bounds for the CRCM model, and analyzed the bounds on the variance of the position parameters (i.e., the diagonal elements of the CRB). We considered only the CRCM model, since its location parameters are physically meaningful: they are the distance from the center, the elevation, and the azimuth limits. On the other hand, the source positions in the VPCM and VPVM models consist of curve parameters and expansion coefficients that do not have such clear physical meaning.

We first investigated the effects of the source length and depth on the estimation performance. We chose a moment density $\boldsymbol{q}=[100,100,100]^{T} \mathrm{nA}$ and set $\rho=0$, since it affects only the source orientation. The CRB results for this case are shown in Fig. 3(a)-(c). Fig. 3(a) shows the CRB for $\varphi_{1}$ when it changes from $5^{\circ}$ to $55^{\circ}$ with $p_{0}=85 \mathrm{~mm}, \xi_{0}=45^{\circ}$, and $\varphi_{2}=60^{\circ}$; Fig. 3(b) for $\varphi_{2}$ when it varies from $25^{\circ}$ to $75^{\circ}$ with $p_{0}=$ $85 \mathrm{~mm}, \xi_{0}=45^{\circ}$, and $\varphi_{1}=20^{\circ}$; and Fig. 3(c) for $p_{0}$ with $\xi_{0}=45^{\circ}, \varphi_{1}=20^{\circ}$, and $\varphi_{2}=60^{\circ}$.

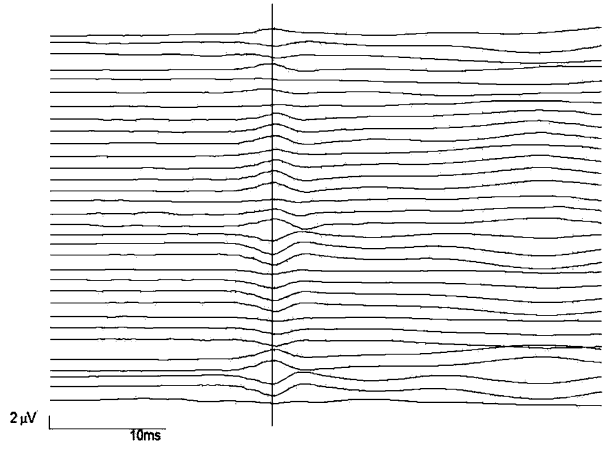

Fig. 4. An example of multichannel EEG recordings of N20 response for a certain subject. The stimulus is applied at $t=0$ and a peak can be clearly seen at $t=20 \mathrm{~ms}$ (indicated by the vertical line).

Then we computed the CRB of $\rho$ in order to see the effect of the orientation of the source position. In this case, we fixed the center of the source at $p_{0}=85 \mathrm{~mm}, \xi=45^{\circ}$, and $\varphi=$ $40^{\circ}$ and rotated the source around its midpoint on the sphere $x^{2}+y^{2}+z^{2}=85^{2}$ with a fixed length of $39 \mathrm{~mm}$. We computed CRBs for different $\rho$ values between -15 and 15 , and the result is shown in Fig. 3(d).

We observe from the CRB values the following.

- Longer sources result in smaller CRBs of the azimuth limits; that is, it is easier to estimate longer sources. Fig. 3(a) and (b) shows that we can estimate $\varphi_{1}$ and $\varphi_{2}$ with standard deviation less than $3^{\circ}$ for a source longer than $12 \mathrm{~mm}$, at a depth of $p_{0}=85 \mathrm{~mm}$ and elevation $\xi=45^{\circ}$. The estimation error increases drastically if the two ends of the line are very close [see the part of the curve when $\varphi_{1}>45^{\circ}$ in Fig. 3(a) or when $\varphi_{2}<35^{\circ}$ in Fig. 3(b)].

- Deeper sources produce larger CRBs of the radius component $p_{0}$. We can infer from Fig. 3(c) that the source depth can be estimated with less than $3 \mathrm{~mm}$ error if the source is more than $70 \mathrm{~mm}$ away from the head center. Therefore, deeper sources result in worse estimation accuracy.

- The CRB is larger for larger slope $\rho$; that is, it is easier to estimate a line source that extends along the azimuth direction.

\section{B. Application to Real EEG Data of N20 Response}

We present results using real EEG measurements of N20 response from four healthy subjects. The N20 generator is known to be along the wall of the central sulcus, which is more extended in one direction (from superior-posterior to inferior-anterior) and is a good example where the line-source models can be applied. The EEG data were recorded over the contralateral somatosensory cortex when square-wave current pulses of $0.2 \mathrm{~ms}$ were delivered to the right or left wrist at a stimulation rate of $4 \mathrm{~Hz}$. The data were sampled at $5000 \mathrm{~Hz}$ with a $1500 \mathrm{~Hz}$ anti-aliasing low-pass filter, resulting in 250 time samples for each subject; see Fig. 4 for an example of N20 response where the stimulus is applied at $t=0$.

For each subject, we picked the time point when the peak occurs (around $t=20 \mathrm{~ms}$ ) and applied the VPVM model to estimate the line-source parameters. We used $\psi(s)=[1, s]^{T}$ 
TABLE III

ESTIMATION PERFORMANCE RESULTS FOR REAL EEG MEASUREMENTS OF N20 RESPONSES

\begin{tabular}{|c|c|c|c|c|}
\hline Subject & A & B & C & D \\
\hline MSE with dipole model $\left(\mu \mathrm{V}^{2}\right)$ & 3.54 & 1.98 & 2.69 & 1.26 \\
MSE with VPVM model $\left(\mu \mathrm{V}^{2}\right)$ & 2.49 & 1.27 & 2.53 & 0.95 \\
Error decreased (\%) & 30 & 36 & 6 & 25 \\
Est. source length (mm) & 19.1 & 21.8 & 7.0 & 15.6 \\
\hline
\end{tabular}

(a)

(b)

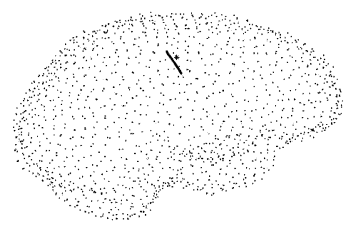

(c)

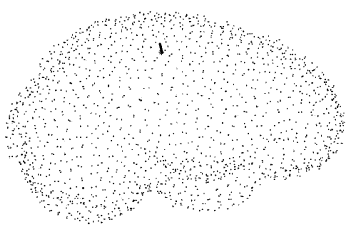

(d)

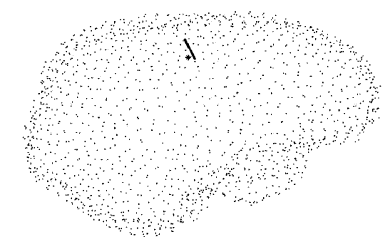

Fig. 5. Estimated line and dipole sources for real EEG measurements of N20 responses. In each plot, the dot (".") represents the nodes on the brain mesh, the star ("*") the estimated dipole source, and the line ("-") the estimated line source.

and $\boldsymbol{\gamma}(s)=\left[1, s, s^{2}\right]^{T}$ as basis functions to represent the source location and its moment density, respectively. In order to initialize the estimate, we first used the dipole source model and located the approximate center of the electrical activities; then we determined the initial values of the VPVM parameters according to this dipole position and estimated its extent. We computed the MSEs for both the dipole and VPVM models and estimated the source length; see Table III. We plot in Fig. 5 the estimated dipole and line-source positions for each subject in the brain meshes obtained from MRI. In Fig. 6, we give the iso-contour maps of the original potential used for estimation (the first column), the residual potential using the dipole model (the second column), and the residual potential using the line-source model (the third column). The residual data are computed by

$$
\operatorname{Residual}(\boldsymbol{y}, \hat{\boldsymbol{y}})=\boldsymbol{y}-\frac{\boldsymbol{y} \cdot \hat{\boldsymbol{y}}}{\hat{\boldsymbol{y}}^{\prime} \hat{\boldsymbol{y}}} \hat{\boldsymbol{y}}
$$

where $\boldsymbol{y}$ is the measured potential and $\hat{y}$ the fitted potential using a certain kind of source model. Clearly, the smaller the residual is, the better the source model fits the data. Comparing the last two columns in Fig. 6, we can see that the line-source model captures more spatial information of the source and clearly reduces the residual potential (see subjects $a, b$, and d). The improvement is not so obvious for the subject $\mathrm{c}$, which may imply that the real source in this subject is more focal than the others. These plots are consistent with the results in Table III where we can see that the VPVM model has a smaller MSEs for all four subjects.

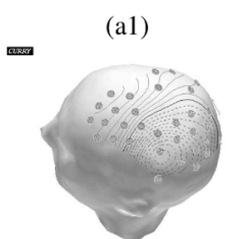

(b1)

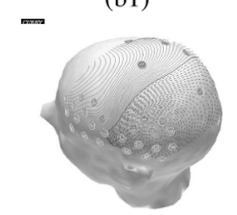

(c1)

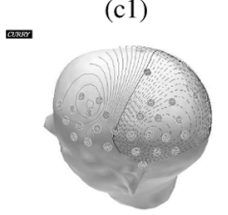

(d1)

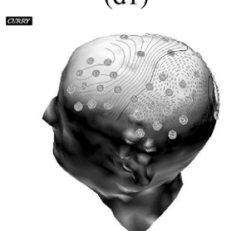

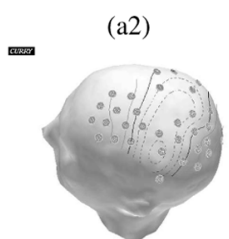

(b2)

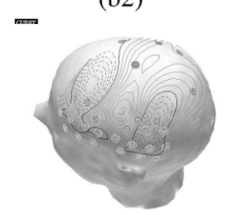

(c2)

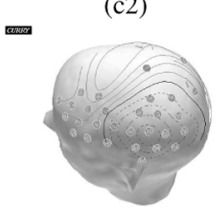

(d2)

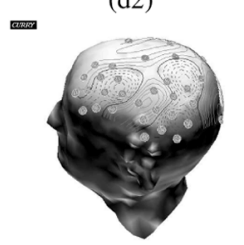

(a3)

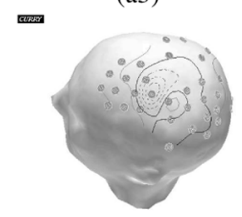

(b3)

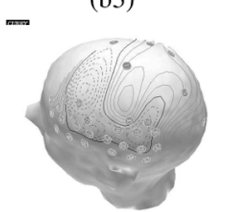

(c3)

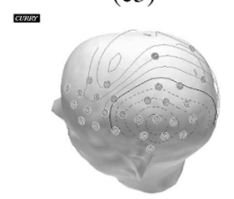

(d3)

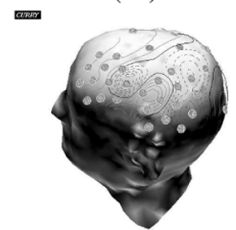

Fig. 6. The iso-contour maps of the original and residual potentials for four different subjects. Each row represents the results for a certain subject (a, b, c, or d) and the corresponding line increment is $100,50,200$, and $100 \mathrm{nV}$, respectively. From left to right, each column represents the original potential, the residual potential using the dipole model, and the residual potential using the line-source model, respectively. The circles indicate the electrode positions.

We mentioned in Section I that other than the choice of source model, the head model is also an important factor affecting the accuracy of the inverse solutions. In our experiments, we used the BEM based realistic head model and all the surfaces were tessellated into triangles with size $7 \mathrm{~mm}$. A previous study by Haueisen et al. [38] showed that: 1) only with a triangle size less than $10 \mathrm{~mm}$ it is possible to achieve stable estimation results; 2) in order to get acceptable errors within the stable region, the ratio between the dipole depth and the triangle size must not be less than 0.5. Both conditions are satisfied in our case, resulting in acceptable errors from head modeling.

\section{DISCUSSION}

We proposed three parametric line-source models for EEG with increasing degrees of freedom. We assumed a three-layer realistic head model and solved the EEG forward problem using the boundary element method. We derived the MLEs and CRBs of the source parameters and evaluated the model fitness using the MSE and AIC values. The main results can be summarized as follows.

- Numerical results show that the proposed line-source models fit better than the dipole source model for extended sources.

- The CRB on the position parameters indicates that longer sources result in better estimation accuracy, deeper sources produce poorer performance, and it is easier to estimate the sources extending along the azimuth direction. 
- The proposed models explain the real EEG measurements of N20 responses (obtained by electric stimulation to the median nerve) better than the dipole source model.

Our method is a useful addition to the current algorithms. It provides a good approximation to the electric sources which are more extended in one dimension (e.g., along the wall of sulcus in the brain surface). Our models differ from the commonly used distributed source imaging approaches in the following aspects. First, the source's spatial extent is directly parameterized and estimated, providing more specific information. Secondly, using the basis function expansion, we can easily incorporate the prior information of the source spatial distribution into the forward model, thus improve the localization accuracy. In this work, we choose polynomials as our basis functions for the reasons that 1) using polynomials is in general a natural choice since enough numbers of polynomials can represent any function to a certain degree of continuation; 2) we know a priori that the source should be in a direction along the central sulcus which goes from superior-posterior to inferior-anterior, hence it suffices to use polynomials to represent it.

We showed using both the simulated data and real EEG measurements that our line-source models provide smaller MSEs than the dipole source model. This improvement may look obvious at a first glance considering that our models are more general and there are more unknown parameters involved. However, the key point is that we are also able to estimate the source extent information using our models, which is a useful property and provides the basis for developing parametric surface-source modeling for EEG. Surface-source modeling will be practically useful, for example, for modeling the cortical generators of scalp EEG interictal spikes in epilepsy. It was recently shown by Tao et al. [39] that in order to produce scalp recognizable potentials, $90 \%$ of the cortical spikes in their study have a source area greater than $10 \mathrm{~cm}^{2}$; and it is common to have synchronous or at least temporally overlapping activation of $10-20 \mathrm{~cm}^{2}$ of gyral cortex. Therefore, it would be interesting to see whether we can use parametric models to represent such an extended source and even to capture the spreading of the source with time. Additionally, we can also consider more complex noise models (e.g., unknown spatially correlated noise) and obtain the MLEs of the unknown parameters using the extended GMANOVA technique as in [4].

\section{REFERENCES}

[1] W. W. Orrison, J. D. Lewine, J. A. Sanders, and M. F. Hartshorne, Functional Brain Imaging. St. Louis, MO: Mosby-Year Book, 1995.

[2] E. Niedermeyer and F. L. Silva, Electroencephalography. Basic Principles, Clinical Applications and Related Fields. Baltimore, MD: Urban and Schwarzenberg, Inc., 1987.

[3] C. M. Michel, M. M. Murray, G. Lantz, S. Gonzalez, L. Spinelli, and R. G. de Peralta, "EEG source imaging," Clin. Neurophysiol., vol. 115, pp. 2195-2222, Oct. 2004.

[4] A. Dogandzic and A. Nehorai, "Estimating evoked dipole responses in unknown spatially correlated noise with EEG/MEG arrays," IEEE Trans. Signal Process., vol. 48, no. 1, pp. 13-25, Jan. 2000.

[5] J. C. Mosher, R. M. Leahy, and P. S. Lewis, "EEG and MEG: Forward solutions for inverse methods," IEEE Trans. Biomed. Eng., vol. 46, no. 3, pp. 245-259, Mar. 1999.

[6] J. C. Mosher, P. S. Lewis, and R. M. Leahy, "Multiple dipole modeling and localizaion from spatio-temporal MEG data," IEEE Trans. Biomed. Eng., vol. 39, no. 6, pp. 541-557, Jun. 1992.
[7] S. Supek and C. J. Aine, "Simulation studies of multiple dipole neuromagnetic source localization: Model order and limits of source resolution," IEEE Tran. Biomed. Eng., vol. 40, no. 6, pp. 529-540, Jun. 1993.

[8] L. Gavit, S. Baillet, J. Mangin, J. Pescatore, and L. Garnero, "A multiresolution framework to MEG/EEG source imaging," IEEE Trans. Biomed. Eng., vol. 48, no. 10, pp. 1080-1087, Oct. 2001.

[9] R. D. Pascula-Marqui, C. M. Michel, and D. Lehmann, "Low resolution electromagnetic tomography: A new method for localizing electrical activity of the brain," Int. J. Phychophysiol., vol. 18, pp. 49-65, Oct. 1994.

[10] J. Sarvas, "Basic mathematical and electromagnetic concepts of biomagnetic inverse problem," Phys. Med. Biol., vol. 32, pp. 11-22, Jan. 1987.

[11] I. Gorodnistky, J. George, and B. Rao, "Neuromagnetic source imaging with focuss: A recursive weighted minimum-norm algorithm," Electroencephalogr. Clin. Neurophysiol., vol. 95, pp. 231-251, Oct. 1995.

[12] P. Valdes-Sosa, F. Marti, F. Gaicia, and R. Casanova, "Variable resolution electric-magnetic tomography," presented at the 10th Int. Conf. Biomagnetism., New York, 2000.

[13] F. N. Wilson and R. H. Bailey, "The electrical field of an eccentric dipole in a homogeneous spherical conducting medium," Circulation, vol. 1, pp. 84-92, 1950.

[14] J. C. De Munck and M. J. Peters, "A fast method to compute the potential in the multisphere model," IEEE Trans. Biomed. Eng., vol. 40, no. 11, pp. 1166-1174, Nov. 1993.

[15] M. Sun, "An efficient algorithm for computation multishell spherical volume conductor models in EEG dipole source localization," IEEE Trans. Biomed. Eng., vol. 44, no. 12, pp. 1243-1253, Dec. 2004.

[16] Z. Zhang, "A fast method to compute surface potentials generated by dipoles within multilayer anisotropic spheres," Phys. Med. Biol., vol. 40, pp. 335-349, Mar. 1995.

[17] T. Kim, Y. Zhou, S. Kim, and M. Singh, "EEG distributed source imaging with a realistic finite-element head model," IEEE Trans. Nucl. Sci., vol. 49, pp. 745-752, Jan. 2002.

[18] C. Muravchik and A. Nehorai, "EEG/MEG error bounds for a static dipole source with a realistic head model," IEEE Trans. Signal Process., vol. 49, no. 3, pp. 470-484, Mar. 2001.

[19] B. N. Cuffin, "Effect of head shape on EEG's and MEG's," IEEE Tran. Biomed. Eng., vol. 37, pp. 44-52, Jan. 1990.

[20] —_, "A method for localizing eeg sources in realistic head models," IEEE Trans. Biomed. Eng., vol. 42, no. 1, pp. 68-71, Jan. 1995.

[21] — - "EEG localization accuracy improvements using realistically shaped head models," IEEE Tran. Biomed. Eng., vol. 43, no. 3, pp. 299-303, Mar. 1996.

[22] G. Nolte and G. Curio, "Current multipole expansion to estimate lateral extent of neuronal activity: A theoretical analysis," IEEE Trans. Biomed. Eng., vol. 47, no. 10, pp. 1347-1355, Oct. 2000.

[23] I. S. Yetik, A. Nehorai, C. Muravchik, and J. Haueisen, "Line-source modeling and estimation with magnetoencephalography," IEEE Trans. Biomed. Eng., vol. 52, no. 5, pp. 839-851, May 2005.

[24] J. C. Mosher, R. M. Leahy, and P. S. Lewis, "Matrix kernels for MEG and EEG source localization and imaging," in Proc. IEEE Int. Conf. Acoust., Speech, and Signal Processing (ICASSP 95), Detroit, MI, May 1995, vol. 5, pp. 2943-2946.

[25] S. M. Kay, Fundamentals of Statistical Signal Processing: Estimation Theory. Upper Saddle River, NJ: PTR Prentice-Hall, 1993.

[26] H. L. Van Trees, Detection, Estimation and Modulation Theory. New York: Wiley, 1968.

[27] H. Akaike, "Information and an extension of the likelihood principle," in Proc. Int. Symp. on Information Theory, Supplement to Problems of Control and Information Theory, Budapest, Hungary, 1973, pp. 267-281.

[28] L. Waldorp, H. Huizenga, A. Nehorai, R. Grasman, and P. Molenaar, "Model selection in spatio-temporal electromagnetic source analysis," IEEE Trans. Biomed. Eng., vol. 52, no. 3, pp. 414-420, Mar. 2005.

[29] C. A. Brebbia and J. Domińguez, Boundary Elements. An Introductory Course, 2nd ed. New York: McGraw-Hill.

[30] S. van den Broek, H. Zhou, and M. Peters, "Computation of neuromagnetic fields using finite-element method and biot-savart law," Med. Biol. Eng. Comput., vol. 34, pp. 21-26, Jan. 1996.

[31] F. Bijma, J. C. de Munck, H. M. Huizenga, R. M. Heethaar, and A. Nehorai, "Simultaneous estimation and testing of sources in multiple MEG data sets," IEEE Trans. Signal Process., vol. 53, no. 9, pp. 11-33, Sep. 2005. 
[32] J. C. de Munck, P. C. M. Vijn, and F. H. L. da Silva, "A random dipole model for spontaneous brain activity," IEEE Tran. Biomed. Eng., vol. 39, no. 8, pp. 791-804, Aug. 1992.

[33] A. S. Ferguson, X. Zhang, and G. Stroink, "A complete linear discretization for calculating the magnetic field using the boundary element method," IEEE Trans. Biomed. Eng., vol. 41, no. 5, pp. 455-460, May 1994.

[34] H. A. Schlitt, L. Heller, R. Aaron, E. Best, and D. M. Ranken, "Evaluation of boundary element methods for the EEG forward problem: Effect of linear interpolation," IEEE Trans. Biomed. Eng., vol. 42, no. 1, pp. 52-58, Jan. 1995.

[35] J. W. Brewer, "Kronecker products and matrix calculus in system theory," IEEE Trans. Circuits Syst., vol. 25, pp. 772-781, Sep. 1978.

[36] D. Gutierrez, A. Nehorai, and C. Muravchik, "Estimating brain conductivities and dipole source signals with EEG arrays," IEEE Trans. Biomed. Eng., vol. 51, no. 12, pp. 2113-2122, Dec. 2004.

[37] H. Buchner, M. Fuchs, H. A. Wischmann, O. Dössel, I. Ludwig, A. Knepper, and P. Berg, "Source analysis of median nerve and finger stimulated somatosensory evoked potentials: Multichannel simultaneous recording of electric and magnetic fields combined with 3D-MR tomography," Brain Topogr., vol. 6, pp. 299-310, 1994.

[38] J. Haueisen, A. Boettner, M. Funke, H. Brauer, and H. Nowak, "The influence of boundary element discretization on the forward and inver problem in electroencephalography and magnetoencephalography," Biomedizinische Technik, vol. 42, pp. 240-248, 1997.

[39] J. X. Tao, A. Ray, S. Hawes-Ebersole, and J. S. Ebersole, "Intracranial EEG substrates of scalp EEG interictal spikes," Epilepsia, vol. 46, pp. 669-676, May 2005.

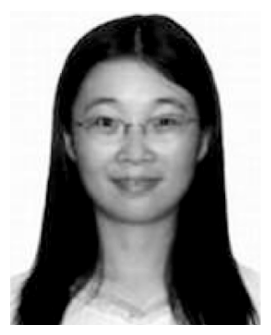

Nannan Cao (S'06) received the B.S. degree in electrical engineering from the University of Science and Technology of China, Hefei, in 2002. She is currently working toward the Ph.D. degree in the Department of Electrical and Systems Engineering, Washington University, St. Louis, MO.

Her research interest is in statistical signal processing with applications to biomedicine.

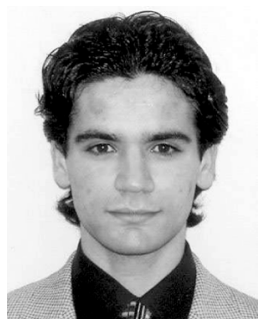

İmam Şamil Yetik (M'05) was born in Istanbul, Turkey, in 1978. He received the B.Sc. degree in electrical and electronics engineering from Bogazici University, Istanbul, in 1998, the M.S. degree in electrical and electronics engineering from Bilkent University, Ankara, Turkey, in 2000, and the Ph.D. degree in electrical and computer engineering from the University of Illinois at Chicago, in 2004.

$\mathrm{He}$ joined the Department of Electrical and Computer Engineering at the Illinois Institute of Technology as an Assistant Professor after his postdoc positions at the University of Illinois at Chicago and University of California at Davis. His research interests are in the areas of biomedical signal and image processing with emphasis on PET image reconstruction, image registration, and EEG/MEG.

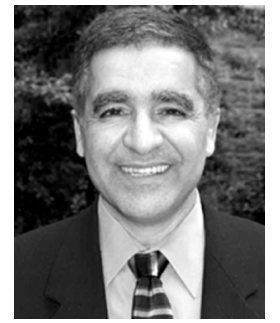

Arye Nehorai (S'80-M'83-SM'90-F'94) received the B.Sc. and M.Sc. degrees in electrical engineering from the Technion, Haifa, Israel, and the Ph.D. degree in electrical engineering from Stanford University, Stanford, CA.

From 1985 to 1995 , he was a faculty member with the Department of Electrical Engineering at Yale University, New Haven, CT. In 1995, he joined the Department of Electrical Engineering and Computer Science at The University of Illinois at Chicago (UIC) as Full Professor. From 2000 to 2001, he was Chair of the department's Electrical and Computer Engineering (ECE) Division, which then became a new department. In 2001, he was named University Scholar of the University of Illinois. In 2006, he assumed the Chairman position of the Department of Electrical and Systems Engineering at Washington University, St. Louis, MO, where he is also the inaugural holder of the Eugene and Martha Lohman Professorship of Electrical Engineering. $\mathrm{He}$ is the Principal Investigator of the new multidisciplinary university research initiative (MURI) project entitled Adaptive Waveform Diversity for Full Spectral Dominance.

Dr. Nehorai was Editor-in-Chief of the IEEE TRANSACTIONS ON SIGNAL PROCESSING during the years 2000 to 2002. In 2003-2005, he was Vice President (Publications) of the IEEE Signal Processing Society, Chair of the Publications Board, member of the Board of Governors, and member of the Executive Committee of this Society. He is the founding editor of the special columns on Leadership Reflections in the IEEE Signal Processing Magazine. He was co-recipient of the IEEE SPS 1989 Senior Award for Best Paper with P. Stoica, co-author of the 2003 Young Author Best Paper Award and co-recipient of the 2004 Magazine Paper Award with A. Dogandzic. He was elected Distinguished Lecturer of the IEEE SPS for the term 2004 to 2005 . He has been a Fellow of the Royal Statistical Society since 1996.

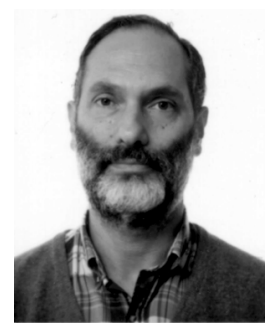

Carlos H. Muravchik (S'81-M'83-SM'99) was born in Argentina, June 11, 1951. He graduated as an Electronics Engineer from the National University of La Plata, La Plata, Argentina, in 1973. He received the M.Sc. in statistics (1983) and the M.Sc. (1980) and $\mathrm{Ph} . \mathrm{D}$. (1983) degrees in electrical engineering, from Stanford University, Stanford, CA.

$\mathrm{He}$ is a Professor at the Department of the Electrical Engineering of the National University of La Plata and a member of its Industrial Electronics, Control and Instrumentation Laboratory (LEICI). He is also a member of the Comision de Investigaciones Cientificas de la Pcia. de Buenos Aires. He was a Visiting Professor at Yale University in 1983 and 1994, and at the University of Illinois at Chicago in 1996, 1997, 1999, and 2003. Since 1999, he is a member of the Advisory Board of the journal Latin American Applied Research. His research interests are in the area of statistical signal and array processing with biomedical, control and communications applications, and nonlinear control systems.

Dr. Muravchik was an Associate Editor of the IEEE TRANSACTIONS ON SigNAL PROCESSING (2003-2006).

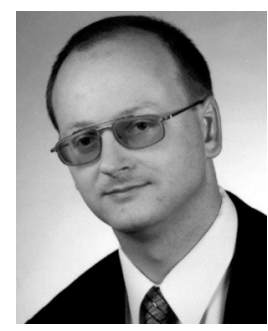

Jens Haueisen (M'02) received the M.S. and Ph.D. degrees in electrical engineering from the Technical University Ilmenau, Ilmenau, Germany, in 1992 and 1996, respectively.

From 1996 to 1998 he worked as a Post-Doc and from 1998 to 2005 as the head of the Biomagnetic Center, Friedrich-Schiller-University, Jena, Germany. Since 2005, he is Professor of Biomedical Engineering and directs the Institute of Biomedical Engineering and Informatics at the Technical University Ilmenau. His research interests are in the numerical computation of bioelectromagnetic fields and the analysis of bioelectromagnetic signals. 